\title{
User Research im Zukunftsorientiertem Design-Thinking: Eine Ganzheitliche Methode für das Stakeholder-Management in der Service-Optimierung
}

Mehdi Mozuni, Maren Ohlhoff, Gerhard Glatzel

User Research ist eine wichtige und in vielen Ansätzen die erste Phase eines iterativen Designprozesses. Aber wie kann eine valide User Research für eine in die weite Zukunft gerichtete Produktgestaltung durchgeführt werden?

User Research erfordert die Einbindung zukünftiger NutzerInnen oder potentieller Kundlnnen. Diese können aber überwiegend keine plausible Auskunft über die eigenen Bedürfnisse der (fernen) Zukunft geben. Auch können sie ausgehend vom individuellen Ist-Zustand nicht zwischen realisierbaren und utopischen Produktkonzepten unterscheiden.

In diesem Beitrag diskutieren wir die Herausforderungen von User Research speziell für mittel- und langfristige Zielsetzungen und stellen einen Lösungsansatz vor: Wir empfehlen daher den Designprozess mit einem Szenarioverfahren zu initiieren, welcher eine Trendanalyse und eine Expertenbefragung beinhaltet. Aufbauend auf den Ergebnissen aus der Szenarioarbeit werden die NutzerInnen in einer späteren Phase des Designprozesses zur Evaluierung von Nutzungsszenarien eingebunden.

Anhand von zwei aktuellen transdisziplinären Projekten „Elektrifizierung von landwirtschaftlichen Betrieben" und "Sustainable Energy-Efficient Aviation", in denen der angenommene Status zukünftiger sozialer, wirtschaftlicher und politischer Trends die Perspektive und das Konsumverhalten fiktiver Nutzerlnnen kontinuierlich prägen wird, zeigen wir, wie wir Nutzerforschung in strategischen Designprozessen mit hoher Vorhersagegüte einsetzen.

Keywords: UX Research, Szenarioprozess, Zukunftsforschung, Design Thinking, Nutzerrecherche, Morphological Delphi 


\section{Entwicklung von Lösungen (Produkt, Service, System) für eine mittel- oder langfristige Perspektive}

Welche nutzerzentrierten digitalen Lösungen und Servicekonzepte könnten aus der Systemgestaltung einer zukünftigen Landwirtschaft, in denen die Agrartechnik zu $100 \%$ elektrifiziert ist abgeleitet werden? Wie wird sich ein europäisches Luftfahrtsystem der ferneren Zukunft aus Nutzersicht entwickeln?

Ob zur Identifizierung von zukünftigen Geschäftsmöglichkeiten oder zur Optimierung ihrer Zukunftstauglichkeit hinsichtlich einer nachhaltigen Entwicklung (Malhotra, Das \& Chariar, 2014: 121), sind viele Regierungen und große Unternehmen zunehmend an einer langfristigen Service- und Produktgestaltung interessiert (siehe z. B. Airbus, 2016, S. 32 ff. Götz, Held, Volland \& Winkler, 2017, S. 71-78).

Eine kritische Voraussetzung für die Bearbeitung solcher Projektvorhaben ist eine nahezu realistische Antizipation der zukünftigen Mensch-System-Interaktion (Human System Interaction - HSI). Es gilt zu evaluieren, ob das neu zu gestaltende System den Wünschen, Bedürfnissen und dem Konsumverhalten der NutzerInnen in einem System von morgen gerecht werden kann.

Unter InnovationsmanagerInnen, HSI-DesignerInnenn und User Experience Researchern (UXR) werden zunehmend verschiedene Ansätze der User Research (UR) verwendet (siehe z. B. Goodman, Kuniavsky \& Moed, 2013, S. 45 ff.; Konstantakis \& Caridakis, 2020, S. 1; Robinson, Lanius \& Weber, 2018, S. 19). Es stellt sich daher die Frage, welche dieser Ansätze und Werkzeuge zuverlässige Ergebnisse für ein HSI-Konzept einer fernen Zukunft liefern können.

\section{Nutzerzentrierung ohne NutzerInnen}

User Research (UXR, auch User Experience Research, UXR, genannt) ist eine wichtige (und in den meisten Ansätzen erste) Phase eines iterativen Designprozesses.

Iterative Umfragen und Anwendungstests mit potenziellen NutzerInnen bieten ein solides und zuverlässiges Verständnis über tatsächliche Bedürfnisse, Wünsche und wahrscheinliche Anreize für die Nutzung des zu entwerfenden Produkts oder der neuen Dienstleistung. Aber wie kann die UXR in einem Design-Thinking-Ansatz durchgeführt werden, wenn der oder die Nutzerln nicht erreichbar und/oder nicht in der Lage ist, seine/ihre Bedürfnisse vorherzusagen oder zu formulieren?

Dieses Problem gilt insbesondere für Dienstleistungen, die eine sehr lange strategische Vorbereitung erfordern, wie z. B. Elektrifizierung landwirtschaftlicher Betriebe. 
Ähnliche Probleme können auch bei der Entwicklung von Produkten mit einem langen Lebenszyklus (z. B. Design von Zivilflugzeugen) oder bei unternehmerischen Fragestellungen beobachtet werden, wenn zum ersten Mal Pionierarbeit für eine Dienstleistung erbracht wird (z. B. Netflix). In all diesen Fällen übersteigen die im System vorhandenen sozio-technischen Eventualitäten die verfügbaren Informationen, denn das Zielsystem ist komplex, unterbestimmt und einem Entwicklerteam fehlt folglich das Wissen, welches für die Entwicklung nachhaltiger, benutzerfreundlicher Produkte und Lösungen erforderlich ist.

Um diesen Herausforderungen zu begegnen, müssen wir die Mensch- System-Interaktion (HSI) jener Zeit und mögliche Entwicklungspfade dahin antizipieren. Das heißt, wir müssen die Wechselwirkungen zwischen NutzerInnen und möglichen Lösungen in einem noch nicht existenten System bewerten. Damit werden wir auf zwei methodische Hindernisse stoßen:

1. NutzerInnen mit einem funktionalen Prototyp (Blomkvist, 2014, S. 27) zu konfrontieren und hinsichtlich der Nutzerfreundlichkeit zu evaluieren ist kaum möglich oder nur mit großem technischem Aufwand realisierbar.

2. "Der/die NutzerIn der Zukunft" existiert noch nicht. Übliche marketinggesteuerte Umfragen und ähnliche Erhebungen bei aktuellen Kunden und Kundinnen können die Wünschbarkeit unserer Lösung nicht bestätigen, da die Nutzerln von heute nicht vollständig die Denkweise und andere soziokulturelle Merkmale der nächsten Generation(en) repräsentieren kann.

Zukunftsforschende sind häufig mit diversen soziotechnischen Unsicherheiten konfrontiert. Daher werden die Wissenslücken mittels der Szenariotechnik mittels Durchführung sogenannter Konsistenzanalysen und Plausibilitätschecks für die zu treffenden Annahmen gefüllt (Ritchey, 1998, 2011). Es ist hervorzuheben, dass Szenarien Wahrscheinlichkeiten und keine Fakten diskutieren (Huss, 1988), ebenso erheben sie keinen Anspruch darauf, reelles Wissen über die Zukunft zu erzeugen (Kosow \& Gaßner, 2008). Ihr Ziel besteht eher darin, durch transdisziplinäre Diskussion und gegenseitige kreative Impulse, Innovationen zu fördern. Damit stellen sich die folgenden zwei Fragen: Wie lässt sich UXR in einem innovativen Diskurs im Rahmen eines Szenarioprozesses integrieren? Welche Methoden und Werkzeuge der UXR sind am besten mit der Szenariotechnik kompatibel?

Mozuni und Jonas (2018) schlagen den Ansatz "Morphological Delphi“ vor, bei dem Werkzeuge der Zukunftsforschung in die Design-Thinking-Methode so integriert werden, dass mithilfe des Konsenswissens von ExpertInnen systematisch Szenarien generiert werden. Daran anknüpfend schlagen wir einen schrittweisen Ansatz vor, bei dem

User Research im Zukunftsorientiertem Design-Thinking: Eine Ganzheitliche Methode für das Stakeholder-Management in der Service-Optimierung 165 
nicht nur das Wissen und die Meinung von ExpertInnen, sondern auch von NutzerInnen effektiv in eine Morphologische Analyse einbezogen werden, beispielsweise mittels quantitativer Umfragen und Interviews.

\section{Befragung von ExpertInnen anstelle von NutzerInnen}

Ein methodisches Problem bei der Produktentwicklung für einen mittel- und langfristigen Zukunftshorizont ist das Unvermögen der NutzerInnen, zukünftige Bedürfnisse darzulegen. Hier wäre zwar eine qualitative User Research (z. B. in Fokusgruppen) effektiver als eine quantitative Forschung (z. B. standardisierte Befragung), doch eine allgemeine Marktforschung oder das Sammeln von Daten durch Befragungen der aktuellen NutzerInnen würde uns irreführende Ergebnisse liefern. Denn viele vermeintliche Wünsche und/oder zukünftige Bedürfnisse könnten auf lange Sicht obsolet werden oder sind den NutzerInnen schlicht noch nicht bekannt. Der Grund dafür ist, dass unbekannte Daten und mehrdeutige Informationen über die Zukunft das bekannte, verständliche Wissen der NutzerInnen überschreiten. In einem solchen Fall, in dem übliche UXR-Methoden keine wegweisenden Erkenntnisse über "den Benutzer der Zukunft“ liefern können, sind qualitative Expertenmeinungen eine geeignete Alternative für ein besseres HSI Verständnis (Everett, 1993; Sackman, 1975). ExpertInnen können politische, wirtschaftliche, technische, ökologische und soziale Faktoren in Beziehung setzen und innovative Szenarien liefern. Szenarien können dann als Prototypen und in gängigen UX-Untersuchungen verwendet werden. Die aktuelle Pandemie liefert viel Anschauungsmaterial, wie Expertenprognosen einer Entwicklung zwar bemerkenswert treffsicher sind, aber das exponentielles Wachstum einer Infektion überfordert das erfahrungsgesteuerte und linear orientierte Handeln der Akteure und verunsichert Betroffene.

\section{Szenarien als Prototypen für UXR}

Wie Mogensen (1994) feststellt, sind Prototypen Repräsentationen davon, wie die Zukunft aussehen sollte oder könnte. Blomkvist (2014) sieht Prototypen ähnlich, und zudem als Stellvertreter für zukünftige Dienstleistungen. Diese Definition findet sich auch in der Zukunftsforschung über Szenarien wieder (siehe z. B. Börjeson et al., 2006; Fink \& Schlake, nd; Malhotra et al., 2014). Zusätzlich zu ihrer Funktion, Diskurse unter ExpertInnen zu ermöglichen, eignen sich Szenarien als effektive Service-Prototypen, die im UXR-Ansätzen Anwendung finden können, beispielsweise als ServiceWalkthrough (Blomkvist \& Bode, 2012, S. 3).

Prototypenentwicklungen von Serviceleistungen unterscheiden sich von der Prototypenentwicklung physischer Produkte, da sie die gesamte Mensch-System-Interaktion 
berücksichtigen müssen, anstatt sich auf Mikrofunktionen zu konzentrieren. Dennoch sind viele DesignerInnen an traditionelle UXR-Ansätze, die auf Mikro- (digitale) Lösungen zugeschnitten sind, gewöhnt und verwenden diese immer noch (ebd. S. 2). Mit Hilfe der Szenariotechnik können User Researcher Szenarien generieren, als Service Walkthroughs visualisieren und diese im Anschluss mit ihren herkömmlichen UXR-Methoden gemeinsam mit den NutzerInnen evaluieren. Abbildung 1 stellt die Einbettung der User Research in einer zukunftsorientierten Produktentwicklung dar: Zunächst werden in einer Vorbereitungsphase drei "Trend/Best/Worst Case“ Szenarien von einem Expertengremium prognostiziert, entwickelt und visualisiert. Diese werden dann durch NutzerInnen iterativ getestet, evaluiert und im Nachhinein als Produkt-, Serviceund Entscheidungsvorschläge herausgegeben. So reduzieren DesignerInnen die Gefahr, die teilnehmenden NutzerInnen mit einer Unmenge von Eventualitäten zu konfrontieren und dadurch die Validierung der Ergebnisse der User Research zu beeinträchtigen.

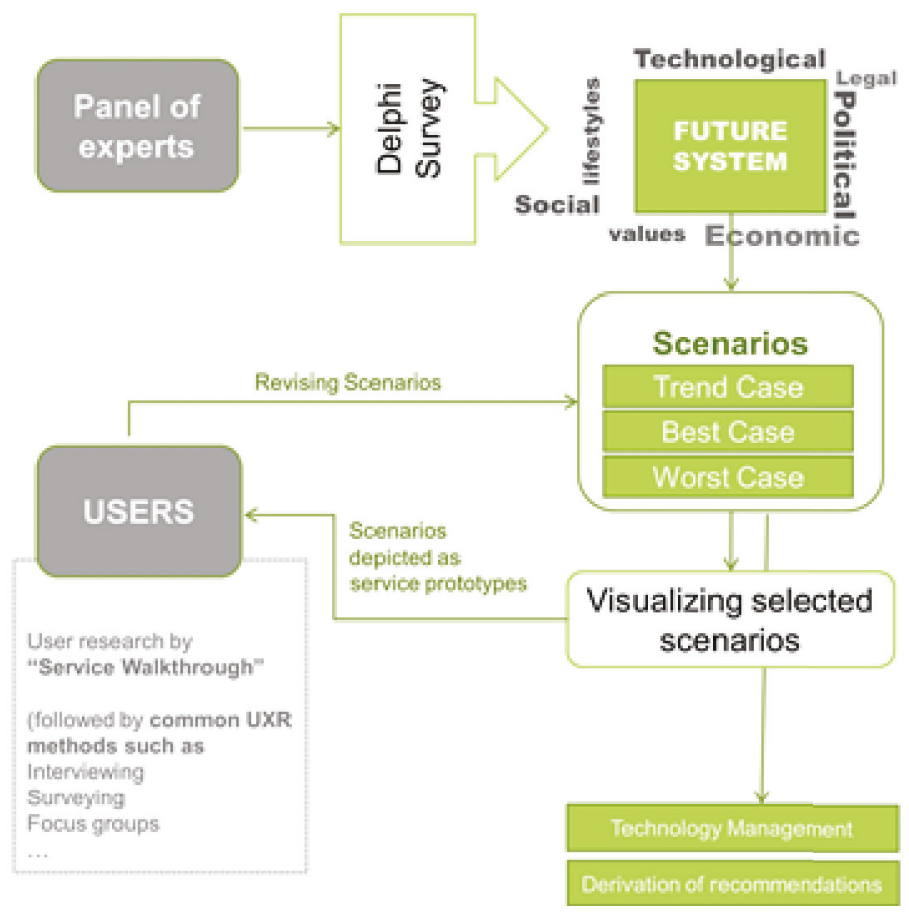

Abbildung 1: Integration von UXR-Werkzeugen in einen Szenarioprozess 


\section{Szenario-Prototyping in der Praxis}

In unseren beiden Forschungsprojekten E4A und SEA haben wir eine morphologische Analyse durchgeführt, um die Mensch-System Interaktionen (HSI) im Kontext des Technologiemanagements aus der Vogelperspektive zu betrachten (Sustainable and energy Efficient Aviation: Gefördert durch die DFG; Projektwebsite: https://www.tubraunschweig.de/se2a; PEST Assessment of Air Transport System in alternative Futures (Scenair); Projektwebsite: https://www.tu-braunschweig.de/en/se2a/research/projects/ica-a/translate-to-english-a11-scenair-2050).

Das Ergebnis dieser morphologischen Analyse sind Szenarien. Wir erwarten von Szenarien in einem HSI-Kontext, dass sie über das reine Prognostizieren (im probabilistischen Sinne) eines zukünftigen Systemzustandes hinausgehen. So sollen die Szenarien Optimierungsmöglichkeiten und darüber hinaus neue Nutzererfahrungen darstellen. Kurz gesagt: Szenarien müssen veranschaulichen, wie die Mensch-System-Interaktion am besten aussehen könnte, nicht wie sie wahrscheinlich aussehen wird!

Diese Szenarien sind kombinierte UX- und Geschäftsmodellvorschläge, die sich mit Anwendungsfällen im mittel- und langfristigen Kontext befassen (d. h. ab 2030). Szenarien erleichtern auch die Art und Weise, wie das Wissen zwischen Innovationsteams und weiteren EntscheidungsträgerInnen konzipiert und übertragen wird (Ghosh, Schilling \& Wicke, 2017, S. 7ff). Szenarien zwingen EntscheidungsträgerInnen, die interne Sichtweise in eine externe Sichtweise umzuwandeln und ihre zukünftige Position in der Branche einzuschätzen (Huss 1988). Für Huss (ebd.) bieten Szenarien Einblicke in die Geschäftsdynamik und unterscheiden sich grundlegend von Prognosen.

\section{Praktische Implementierung in zwei Forschungsprojekten}

Anhand von zwei laufenden zukunftsorientierten Projekten E4A und $\mathrm{SE}^{2} \mathrm{~A}$ skizzieren wir das Verfahren zur Integration von User Research in die Untersuchung zukünftiger Systemzustände. Beide Projekte zeichnen sich durch folgende Merkmale aus:

- Adressierung eines zukünftigen Systems

- Notwendigkeit der Ableitung zukünftiger, digitaler und analoger Produkte

- Herausforderung im transdisziplinären Technologiemanagement

- HSI-Management mit Nutzerzentrierung

— Für die EntscheidungsträgerInnen der Politik müssen Handlungsempfehlungen generiert werden 


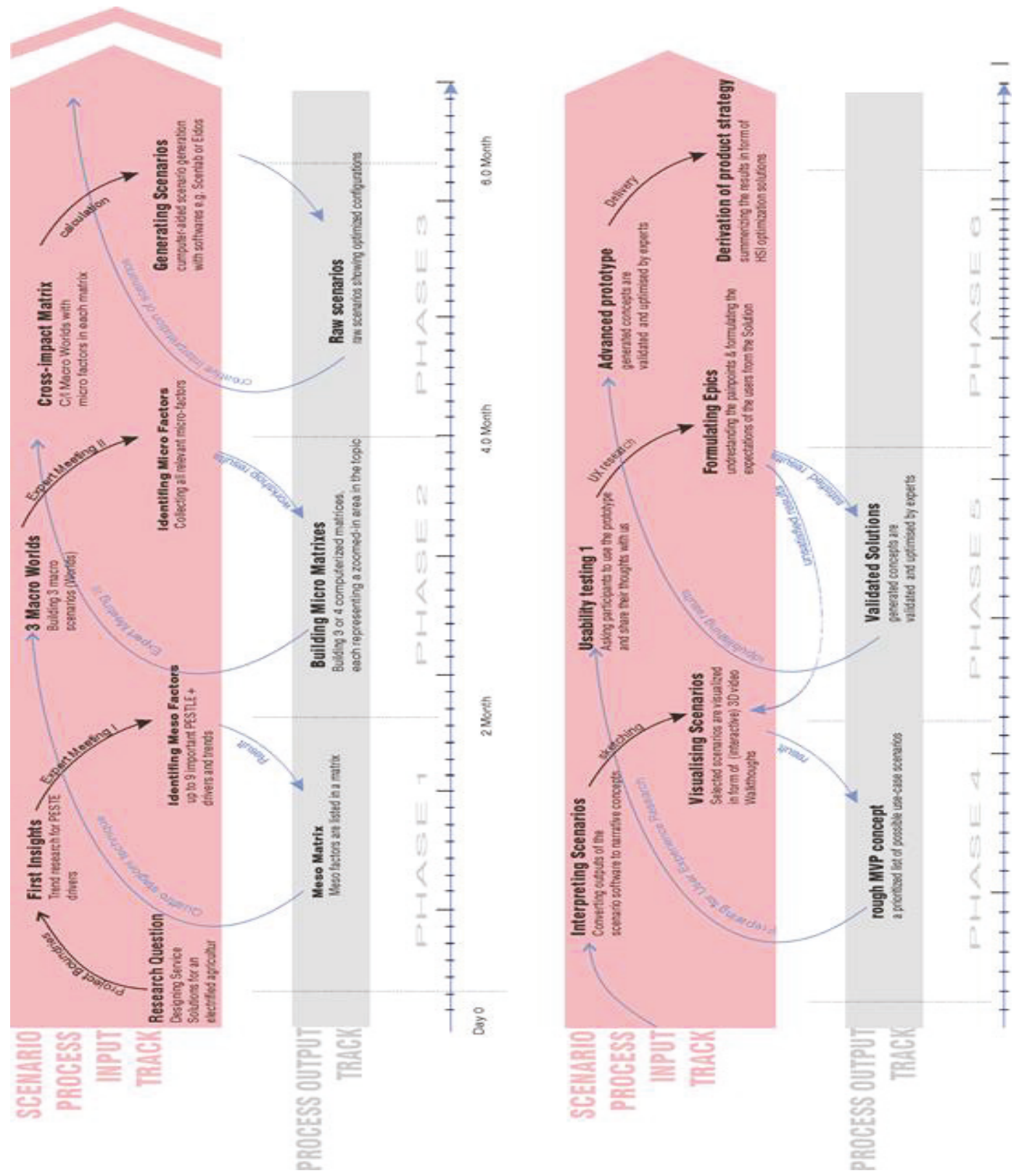

Abbildung 2: Eine generische Iteration für den Morphological Delphi-Prozess

User Research im Zukunftsorientiertem Design-Thinking: Eine Ganzheitliche Methode für das Stakeholder-Management in der Service-Optimierung 169 
Zum besseren Verständnis für einzelne Schritte folgen wir einer umgekehrten zielorientierten Anforderungschronologie (Van Lamsweerde, 2001, S. 7):

1. Ziel a: Für die Gestaltung im politischen Raum müssen Empfehlungen generiert werden Ziel b: Nachhaltige Nutzerzentrierte Optimierung der HSI Voraussetzung: Vorherige Evaluierung durch NutzerInnen

2. Ziel: Bewertung der Lösungen durch NutzerInnen Voraussetzung: User Research z.B. anhand von Service Walkthroughs

3. Ziel: Bereitstellung und Ausführung von Service Walkthroughs Voraussetzung: Szenarien müssen narrativ ausgearbeitet und visualisiert werden (z.B. über VR-Prototyping)

4. Ziel: Generierung von Szenarien Voraussetzung a: Durchführung einer morphologischen Analyse (Álvarez \& Ritchey, 2015, S.1) Voraussetzung b: Es müssen transdisziplinäre Delphi-Workshops durchgeführt werden

5. Ziel a: Durchführung einer morphologischen Analyse Ziel b: Einbezug von quantitativem und qualitativem Wissen aus verschiedenen PESTE-Disziplinen Voraussetzung: Cross-Impact-Analyse und Konsistenzanalyse (Mozuni \& Jonas, 2018b)

6. Ziel: Durchführung einer Cross-Impact-Analyse (siehe auch Ohlhoff, Mozuni, Glatzel 2021 in diesem Band) Voraussetzung : Erstellen einer Matrix bestehend aus Schlüsselfaktoren (Spalten) und ihren möglichen Projektionen (Zeilen); Auflisten von (bis zu vier) Makrowelten als Projektionen einer Spalte und mehreren PESTE-Mikrofaktoren (Empfehlung: bis zu 9 Faktoren) mit jeweils mehreren alternativen Projektionen in weiteren Spalten

7. Ziel: Aufbau von Makrowelten Voraussetzung : Erstellen von drei oder vier Makroszenarien -welten mit Hilfe der Quattro-Stagioni-Szenariotechnik (Godet, 2006, S. 82)

8. Ziel: Quattro Stagioni-Szenariotechnik Voraussetzung : Sensitivitätsanalyse (nach Vester, 2002)

9. Ziel: Durchführung einer Sensitivitätsanalyse Voraussetzung: Durchführung von disziplinübergreifenden Delphi-Workshops zur Ermittlung der zwei aktivsten Faktoren im antizipierten System

10. Ziel: interdisziplinäre Delphi-Workshops Voraussetzung a: Sammeln von quantitativem und qualitativem Wissen aus verschiedenen PESTE-Disziplinen 
Voraussetzung b: Recherche und Einigung über Anzahl, Formulierung und Beschreibung der Schlüsselfaktoren (Empfehlung: bis zu 10 Faktoren) und ihrer möglichen Projektionen (Empfehlung: Pro Faktor max. 4 Projektionen)

Dieser Prozess erfolgt in mehreren iterativen Schritten und kann gegebenenfalls einem agilen Prozessablauf im kommerziellen Projektmanagement angepasst werden. Abbildung 2 zeigt eine allgemeine Iteration, in einem hypothetischen zwölfmonatigen Projektzeitplan, für die oben beschriebenen zehn Schritte: von der Definition der Forschungsfrage über die Gewinnung erster Erkenntnisse bis hin zur Erstellung von Roadmaps und der Vermittlung von Ergebnissen. Die Phasen und Schritte können je nach Forschungsprojekt individuell angepasst werden.

Tabelle 1: Anwendung von Methoden in einem zukunftsorientierten morphologischen Delphi

\begin{tabular}{lllll}
\hline Direkte Projektziele & Szenariotechnik & $\begin{array}{l}\text { Delphi- } \\
\text { methode }\end{array}$ & $\begin{array}{l}\text { Morphologische } \\
\text { Analyse }\end{array}$ & $\begin{array}{l}\text { Service Proto- } \\
\text { typing }\end{array}$ \\
\hline $\begin{array}{l}\text { Generierung neuartiger Lö- } \\
\text { sungen }\end{array}$ & + & + & ++ \\
$\begin{array}{l}\text { Vorantreiben der Zusam- } \\
\text { menarbeit (Ritchey 2011a) }\end{array}$ & ++ & ++ \\
$\begin{array}{l}\text { Technologiemanagement } \\
\text { und Entscheidungsfindung }\end{array}$ & ++ & + \\
$\begin{array}{l}\text { Erfassung komplexer Sys- } \\
\text { teme }\end{array}$ & + & & ++ \\
Betrachtung der Zukunft & ++ & & + & ++ \\
\hline
\end{tabular}

\section{Wie geht es weiter?}

Wir nutzen das Szenariodesign als innovatives Untersuchungsinstrument zukünftiger Lösungen und Technologien zur Erreichung gewünschter Zustände. Die nach unseren Vorschlägen designten Szenarien erweitern Möglichkeitsräume maximal und liefern Voraussetzungen dafür, wie wir diese Möglichkeitsräume optimal nutzen können. Die dafür eingesetzte User Researches steht in engem Zusammenhang mit beinflussbaren und nicht beeinflussbaren PESTE-Variablen. Für die angeführten Forschungsvorhaben haben wir sehr gut einsetzbare Expertenpools, die wir für eine inhaltliche Arbeit mit den geschilderten Ansätzen nutzen werden, entwickelt. Diese Methoden der Zukunftsforschung werden wir weiterentwickeln. 


\section{Literaturverzeichnis}

ACARE. (2011). Flightpath 2050. Isbn: 978-92-79-19724-6. https://doi.org/10.2777/50266

Airbus. (2016). Mapping Demand. Global Market Forecasts. Zugriff am 24.1.2018. Verfügbar unter:

http://www.team.aero/files/airbusforecast/Airbus-GMF-2016-2035-MappingDemand-full_book.pdf

Álvarez, A. \& Ritchey, T. (2015). Applications of General Morphological Analysis. Acta Morphologica Generalis, 4(1), 1-40.

Becker, A. \& Tay, G. (2017). Automation in Commercial Aviation 2030+. Zugriff am 24.1.2018. Verfügbar unter:

https://www.lls.mw.tum.de/fileadmin/w00bdw/www/Vorlesungen/Handout_Abschlusspraesentation_WS1617.pdf

Blomkvist, J. (2014). Representing Future Situations of Service. Prototyping in Service Design. Verfügbar unter:

http://urn.kb.se/resolve?urn=urn:nbn:se:liu:diva-105499

Blomkvist, J. \& Bode, A. (2012). Using Service Walkthroughs to Co-Create Whole Service Experiences: A Prototyping Technique for Service Design. Proceedings of the 3rd International Service Innovation Design Conference, Holistic Service Innovation \& Co-Creation Experience.

Börjeson, L., Höjer, M., Dreborg, K.-H., Ekvall, T. \& Finnveden, G. (2006). Scenario types and techniques: Towards a user's guide. Futures, 38(7), 723-739. https://doi.org/10.1016/j.futures.2005.12.002

Everett, A. (1993). Piercing the veil of the future. A review of the Delphi method of research. Professional nurse (London, England), 9(3), 181-185.

Fink, A. \& Schlake, 0. (0. J.). Scenario Management—An Approach for Strategic Foresight.

Fresco, J., Meadows, R., Schilling, B. \& Doll, S. (2010). Designing the Future, 902-918. https://doi.org/10.3390/su2040902

Ghosh, R., Schilling, T. \& Wicke, K. (2017). Theoretical framework of systems design for the air transportation system including an inherently quantitative philosophy of scenario development. Journal of Air Transport Management, 58, 58-67. https://doi.org/10.1016/j.jairtraman.2016.09.007

Godet, M. (2006). Strategic Foresight Problems and Methods. Cahiers du Lipsor (Issue n²0.). Paris. Zugriff am 5.9.2016. Verfügbar unter: http://www.laprospective.fr/dyn/francais/memoire/strategicforesight.pdf

Goodman, E., Kuniavsky, M. \& Moed, A. (2013). Observing the User Experience: A Practitioner's Guide to User Research (Second Edition). IEEE Transactions on Professional Communication. https://doi.org/10.1109/tpc.2013.2274110

Götz, C., Held, T., Volland, J. \& Winkler, R. (2017). In der Zukunft ankommen - die nächste Generation von User Research. Wie stellen sich Benutzer die Business-Software der Zukunft vor? Mensch und Computer 2017, (10.-13. September 2017), 71-79. Gesellschaft für Informatik e. V. und die German UPA e. V. 2017 in S. Hess, H. Fischer (Hrsg.).

https://doi.org/10.18420/muc2017-up-0210

Huss, W. R. (1988). A move toward scenario analysis. International Journal of Forecasting, 4(3), 377-388. https://doi.org/10.1016/0169-2070(88)90105-7

De Jong, S., Hoefnagels, R., Slade, R., Faaij, A. \& Junginger, M. (2017). Renewable Jet Fuel in the European Union Scenarios and Preconditions for Renewable Jet Fuel Deployment towards 2030, 34.

Konstantakis, M. \& Caridakis, G. (2020). Adding culture to UX: UX research methodologies and applications in cultural heritage. Journal on Computing and Cultural Heritage. https://doi.org/10.1145/3354002 
Kosow, H. \& Gaßner, R. (2008). Methods of Future and Scenario Analysis - Overview, assessment, and selection criteria. Zugriff am 28.3.2021. Verfügbar unter: https://www.researchgate.net/publication/258510126_Methods_of_Future_and_Scenario_Analysis_Overview_Assessment_and_Selection_Criteria

Van Lamsweerde, A. (2001). Goal-oriented requirements engineering: A guided tour. Proceedings of the IEEE International Conference on Requirements Engineering. https://doi.org/10.1109/isre.2001.948567

Malhotra, S., Das, L. K. \& Chariar, V. M. (2014). Design Research Methods for Future Mapping. International Conferences on Educational Technologies 2014 and Sustainability, Technology and Education 2014 (S. 121-130). aipeh City, Taiwan: International Association for Development of the Information Society. Zugriff am 31.5.2016. Verfügbar unter: http://files.eric.ed.gov/fulltext/ED557342.pdf

Mogensen, P. H. (1994). Challenging Practice: an approach to Cooperative Analysis. DAIMI Report Series. https://doi.org/10.7146/dpb.v23i465.6938

Mozuni, M. \& Jonas, W. (2018a). An Introduction to the Morphological Delphi Method for Design: A Tool for Future-Oriented Design Research. She ji: The Journal of Design, Economics, and Innovation, 3(4), 303-318. Elsevier.

https://doi.org/10.1016/J.SHEJI.2018.02.004

Mozuni, M. \& Jonas, W. (2018b). An Introduction to the Morphological Delphi Method for Design: A Tool for Future-Oriented Design Research. She ji: The Journal of Design, Economics, and Innovation, 3(4), 303-318. Elsevier.

https://doi.org/10.1016/J.SHEJI.2018.02.004

Ritchey, T. (1998). General Morphological Analysis * A general method for non-quantified modelling. Swedish Morphological Society. https://doi.org/citeulike-article-id:8916360

Ritchey, T. (2011). Modeling Alternative Futures with General Morphological Analysis. World Future Review, 3(1), 83-94. https://doi.org/10.1177/194675671100300105

Robinson, J., Lanius, C. \& Weber, R. (2018). The past, present, and future of UX empirical research. Communication Design Quarterly. https://doi.org/10.1145/3188173.3188175

Sackman, H. (1975). Summary evaluation of Delphi. Policy Analysis, 1(4), 693-718. https://doi.org/10.1007/s13398-0140173-7.2

Vester, F. (2002). Die Kunst vernetzt zu denken. Ideen und Werkzeuge für einen neuen Umgang mit Komplexität ; ein Bericht an den Club of Rome. dtv. 


\section{Kontakt}

Prof. Dr.-Ing. Gerhard Glatzel

Dr. Mehdi Mozuni

Maren Ohlhoff

Institut für Designforschung

Hochschule für Bildende Künste Braunschweig

Johannes-Selenka-Platz 1

38118 Braunschweig

g.glatzel@hbk-bs.de

m.mozuni@hbk-bs.de

m.ohlhoff@hbk-bs.de 